share the same entry receptors for some respiratory viruses, ${ }^{5}$ including angiotensin-converting enzyme 2 (ACE2) for SARS-CoV-1 and SARS-CoV-2. ${ }^{2,5}$ In addition, SARS-CoV-2 was detectable in several nasolacrimal system-associated tissues, including the conjunctiva, lacrimal gland, nasal cavity, and throat, thus validating the anatomical bridge between ocular mucosa and the respiratory tract. ${ }^{8}$ Finally, macaques were susceptable to SARS$\mathrm{CoV}-2$ infection via the conjunctival route and progressed to lung infections suggesting the biological importance of eye infection. ${ }^{10}$

Given that SARS-CoV-2 can be transmitted by fomites and droplets that contact the mucous membranes of the mouth and nose, as well as the eyes, it appears that until proven otherwise, HCWs and at-risk citizens in the community should use barriers to protect their entire face including their eyes. Current public health guidance recommends cotton face masks, but given the potential role of the conjunctival route, face shields that provide barrier protection for the entire face might be the superior option. Further research in this area is critically needed.

\section{Acknowledgments.}

Financial support. All authors report no source of funding or support.

Conflicts of interest. All authors report no conflicts of interest relevant to this article.

\section{References}

1. Perencevich EN, Diekema DJ, Edmond MB. Moving personal protective equipment into the community: face shields and containment of COVID-19. JAMA 2020;323:2252-2253.
2. Khunti $\mathrm{K}$, Greenhalgh $\mathrm{T}$, Chan $\mathrm{XH}$, et al. What is the efficacy of eye protection equipment compared to no eye protection equipment in preventing transmission of COVID-19-type respiratory illness in primary and community care? The Centre for Evidence-Based Medicine website. https://www.cebm.net/covid-19/what-is-the-efficacy-of-eye-protectionequipment-compared-to-no-eye-protection-equipment-in-preventingtransmission-of-covid-19-type-respiratory-illnesses-in-primary-andcommunity-care/. Accessed May 28, 2020.

3. Chou R, Dana T, Buckley DI, Selph S, Fu R, Totten AM. Epidemiology of and risk factors for coronavirus infection in healthcare workers. Ann Intern Med 2020 May 5 [Epub ahead of print]. doi: 10.7326/M201632.

4. Fisher DA, Chew MN, Lim YT, Tambyah PA. Preventing local transmission of SARS: lessons from Singapore. Med J Aust 2003;178:555-558.

5. Sun CB, Wang YY, Liu GH, Liu Z. Role of the eye in transmitting human coronavirus: what we know and what we do not know. Front Public Health 2020;8:155.

6. Raboud J, Shigayeva A, McGeer A, et al. Risk factors for SARS transmission from patients requiring intubation: a multicentre investigation in Toronto, Canada. PLoS One 2010;5(5):e10717.

7. Park BJ, Peck AJ, Kuehnert MJ, et al. Lack of SARS transmission among healthcare workers, United States. Emerg Infect Dis 2004;10:244-248.

8. Siedlecki J, Brantl V, Schworm B, et al. COVID-19: ophthalmological aspects of the SARS-CoV 2 global pandemic. Klin Monbl Augenheilkd 2020;237:675-680.

9. Chen L, Liu M, Zhang Z, et al. Ocular manifestations of a hospitalised patient with confirmed 2019 novel coronavirus disease. Br J Ophthalmol 2020 Apr 7 [Epub ahead of print]. doi: 10.1136/bjophthalmol2020-316304.

10. Deng W, Bao L, Gao H, et al. Ocular conjunctival inoculation of SARS-CoV-2 can cause mild COVID-19 in Rhesus macaques. bioRxiv; 2020. doi: 10.1101/ 2020.03.13.990036.

\title{
Limited impact of selective susceptibility reporting of Escherichia coli and Klebsiella isolates from concurrent blood and urine cultures
}

\author{
Brenton C. Hall PharmD ${ }^{1, *}$, Julie S. Alexander DO ${ }^{2, \star}$, Shelby S. Anderson PharmD ${ }^{1}$, Jessica K. Ortwine PharmD, BCIDP ${ }^{1,2}$, \\ Norman S. Mang PharmD, BCIDP ${ }^{1,2}$, Wenjing Wei PharmD, BCPS ${ }^{1,2}$, Linda S. Hynan PhD ${ }^{3}$ and Bonnie C. Prokesch MD² (1) \\ ${ }^{1}$ Department of Pharmacy, Parkland Health \& Hospital System, Dallas, Texas, ${ }^{2}$ Department of Medicine, University of Texas Southwestern Medical Center, Dallas, \\ Texas and ${ }^{3}$ Department of Clinical Sciences, University of Texas Southwestern Medical Center, Dallas, Texas
}

To the Editor-In 2019, the Centers for Disease Control and Prevention reported that $>2.8$ million antibiotic-resistant infections occur in the United States annually, with nearly 35,000 deaths as a result. ${ }^{1}$ One method of minimizing the emergence of antimicrobial resistance is through antimicrobial stewardship. The Infectious Diseases Society of America has published guidelines on antimicrobial stewardship identifying potential interventions to guide appropriate antimicrobial prescribing, which includes selective or cascade reporting of antibiotic susceptibility data. ${ }^{2}$ In cascade reporting, specific antibiotics in the susceptibility report

Author for correspondence: Bonnie C. Prokesch, E-mail: bonnie.prokesch@ utsouthwestern.edu

${ }^{*}$ Authors of equal contribution.

Cite this article: Hall BC, et al. (2021). Limited impact of selective susceptibility reporting of Escherichia coli and Klebsiella isolates from concurrent blood and urine cultures. Infection Control \& Hospital Epidemiology, 42: 647-648, https://doi.org/ $10.1017 /$ ice. 2020.308 are deliberately withheld from the view of clinicians when the organism is susceptible to more narrow-spectrum agents. ${ }^{3}$ Because of the limited data on clinical outcomes, cascade reporting is classified as a weak recommendation. ${ }^{2}$

In 2016, antimicrobial data at our 862-bed county hospital in Dallas, Texas, revealed piperacillin-tazobactam (PT) as the most utilized broad-spectrum gram-negative antimicrobial, with an average of 103 days of therapy per 1,000 patient days. During the same time, 11,306 isolates from the Enterobacterales family were identified from various sources, with $85 \%$ being Escherichia coli or Klebsiella spp. The objective of this retrospective study was to determine whether a cascade reporting system influenced the de-escalation of empiric PT in patients with E. coli and Klebsiella bacteremia due to a urinary source and subsequent effects on patient outcomes.

On September 7, 2017, the clinical microbiology laboratory implemented a cascading antibiotic algorithm for non-extendedspectrum $\beta$-lactamase-producing E. coli and Klebsiella isolates 
from blood and urine cultures. Susceptibility data for PT was suppressed if the organism was nonsusceptible to ceftriaxone. Isolates were considered susceptible to ceftriaxone at a minimum inhibitory concentration of $\leq 1 \mu \mathrm{g} / \mathrm{mL}$, based on Clinical and Laboratory Standards Institute (CLSI) break points. Antibiotic and microbiologic data from September 7, 2016, through September 7, 2018, were retrospectively reviewed. Patients were included if they were at least 18 years old, received empiric PT, and had a monomicrobial non-ESBL E. coli or Klebsiella bacteremia with a concordant monomicrobial urine culture positive for the same organism collected within 24 hours of each other.

The primary end point was de-escalation from PT to a narrower agent, with the hypothesis that a greater percentage of patients would be de-escalated after the intervention. Narrower agents were defined as any narrower-spectrum $\beta$-lactam, ciprofloxacin, or sulfamethoxazole/trimethoprim. De-escalation was further stratified as early or late, if de-escalation occurred after release of either the blood or urine culture susceptibility or after release of both blood and urine cultures susceptibilities, respectively.

Of the 197 patients screened with E. coli or Klebsiella in blood and urine cultures receiving PT therapy during the study period, 103 met study criteria. The preimplementation group had 50 patients and the postimplementation group had 53 patients. Baseline characteristics of the study population were well matched regarding age, race, and gender. A similar percentage of patients were de-escalated in both groups: 45 (90\%) versus 45 (85\%) $(P=.56)$. The most common reason identified for not de-escalating therapy was provider preference based on the patient's clinical status. Ceftriaxone was the most utilized agent for initial de-escalation, followed by ciprofloxacin and sulfamethoxazoletrimethoprim. Empiric PT therapy was utilized for similar median durations in each group (46.2 vs 44.8 hours; $P=.796$ ). Both cohorts displayed rapid de-escalation of antibiotics once susceptibilities were available. Therapy was de-escalated a median of 5.6 hours after the release of susceptibility results in the preintervention group and 4.8 hours in the postintervention group $(P=.506)$. Rates of early de-escalation were similar between both groups: $20(44.4 \%)$ versus $22(48.9 \%)(P=.673)$. Patients in the preintervention group had longer length of stays, although this was not statistically significant ( 6 vs 5 days; $P=.058$ ). The difference in rates of acute kidney injury [3(6\%) vs $9(20 \%)$ ] and C. difficile infection (CDI) [1 (2\%) vs 0] were not statistically significant.

The impact of cascaded susceptibility reporting has been evaluated and has proven useful on several occasions, ${ }^{4-6}$ but only 1 previous study has included frequency of de-escalation as the primary outcome. Johnson et al evaluated the impact of a cascaded susceptibility report on de-escalation from a broad-spectrum $\beta$-lactam to a narrower agent for gram-negative bacteremia. The cascade resulted in more patients being de-escalated to a narrower agent [15 $(48 \%)$ vs $30(71 \%) ; P=.43]$, although this was not statistically significant and did not influence length of stay, CDI, or reinitiation of a broad-spectrum agent within 7 days. ${ }^{5}$
In our study, we found no significant difference in prescribing patterns after the implementation of cascade susceptibility reporting for Enterobacterales. De-escalation was observed in nearly $90 \%$ of patients in both pre- and postintervention groups, and median times to de-escalation were similar in both cohorts.

Because this was a retrospective study focusing on monomicrobial bacteremia secondary to a urinary source, providers may have felt more comfortable in quickly de-escalating, especially after urine culture results were available. Moreover, the overall sample size was small, due to a specific patient population that was included. Finally, providers were allowed to contact the clinical microbiology laboratory for release of suppressed antibiotic results.

Although we observed no difference, we believe that cascade reporting remains a viable intervention that could be added to an program's arsenal; there may be benefit in patient populations not explored in our study. Additionally, this intervention requires little long-term maintenance, and no negative impact was observed. Further research is needed to better identify patient populations most impacted by a cascade algorithm and its overall effectiveness as a stewardship tool.

Acknowledgments. The authors acknowledge Tamara P. Guzman, MT(ASCP) and Donna L Gaffney, MT(ASCP)SM, and Debra Grant, MT(ASCP)SM, for their assistance with microbiology laboratory data and in building the cascade logic, respectively.

Financial support. No funding was received for this study.

Conflicts of interest. All authors report no conflicts of interest related to this article.

\section{References}

1. Antibiotic resistance threats in the United States, 2019. Centers for Disease Control and Prevention website. https://www.cdc.gov/drugresistance/pdf/ threats-report/2019-ar-threats-report-508.pdf. Published 2019. Accessed June 30, 2020.

2. Barlam TF, Cosgrove SE, Abbo LM, et al. Implementing an antibiotic stewardship program: guidelines by the Infectious Diseases Society of America and the Society for Healthcare Epidemiology of America. Clin Infect Dis 2016;62:e51-e77.

3. Coupat C, Pradier C, Degand N, et al. Selective reporting of antibiotic susceptibility data improves the appropriateness of intended antibiotic prescriptions in urinary tract infections: a case-vignette randomised study. Eur J Clin Microbiol Infect Dis 2013;32:627-636.

4. Liao S, Rhodes J, Jandarov R, DeVore Z, Sopirala, MM. Out of sight-out of mind: impact of cascade reporting on antimicrobial usage. Open Forum Infect Dis 2020;7(2):ofaa002.

5. Johnson LS, Patel D, King EA, Maslow JN. Impact of microbiology cascade reporting on antibiotic de-escalation in cefazolin-susceptible gram-negative bacteria. Eur J Clin Microbiol Infect Dis 2016;35:1151-1157.

6. Langford BJ, Seah J, Chan A, et al. Antimicrobial stewardship in the microbiology laboratory: impact of selective susceptibility reporting on ciprofloxacin utilization and susceptibility of gram-negative isolates to ciprofloxacin in a hospital setting. J Clin Microbiol 2016; 54:2343-2347. 